\title{
Laparoscopic distal gastrectomy with D2 lymphadenectomy followed by intracorporeal gastroduodenostomy for advanced gastric cancer: technical guide and tips
}

\author{
Sang-Woong Lee ${ }^{1}$, Masaru Kawai ${ }^{1}$, Keitaro Tashiro ${ }^{1}$, George Bouras ${ }^{2}$, Satoshi Kawashima ${ }^{1}$, Ryo Tanaka ${ }^{1}$, \\ Eiji Nomura $^{3}$, Kazuhisa Uchiyama ${ }^{1}$ \\ ${ }^{1}$ Department of General and Gastroenterological Surgery, Osaka Medical College, Takatsuki, Japan; ${ }^{2}$ Department of Surgery and Cancer, Imperial \\ College London, London, UK; ${ }^{3}$ Department of Gastroenterological and General Surgery, Tokai University Hachioji Hospital, Tokyo, Japan \\ Contributions: (I) Conception and design: SW Lee, M Kawai, E Nomura; (II) Administrative support: M Kawai, K Tashiro, R Tanaka, K Uchiyama; (III) \\ Provision of study materials or patients: SW Lee, M Kawai, K Tashiro; (IV) Collection and assembly of data: SW Lee, M Kawai, R Tanaka; (V) Data \\ analysis and interpretation: SW Lee; (VI) Manuscript writing: All authors; (VII) Final approval of manuscript: All authors. \\ Correspondence to: Sang-Woong Lee. Department of General and Gastroenterological Surgery, Osaka Medical College, 2-7 Daigaku-Machi, \\ Takatsuki, Osaka 569-8686, Japan. Email: sur079@osaka-med.ac.jp.
}

\begin{abstract}
In 1994, Kitano and colleagues first reported laparoscopy-assisted Billroth I gastrectomy. Since then, laparoscopic gastrectomy (LG) has been associated with earlier patient recovery compared with open surgery, and has gained increasing international acceptance. Japan Society of Endoscopic Surgery biennial surveys confirm the increasing use of laparoscopic procedures for treatment of gastric cancer in Japan. Its thirteenth national survey indicates that of 31,264 patients treated at Japanese institutions in 2015, approximately 9,500 (30.3\%) underwent LG, and laparoscopic distal gastrectomy (LDG) was the procedure most commonly performed. Despite evidence supporting the efficacy of LDG for gastric cancer in the short term, however, uncertainty remains concerning the efficacy of LG. Today, phase III randomized control trials on this procedure are ongoing in East Asian countries. Distal gastrectomy (DG) is the most commonly performed mode of resection, and as appropriate surgical techniques need to be acquired by gastric surgeons, here we describe a 'gold standard' method to perform total LDG.
\end{abstract}

Keywords: Gastric cancer; laparoscopic gastrectomy (LG); distal gastrectomy (DG); Billroth I reconstruction; intracorporeal anastomosis

Received: 31 July 2017; Accepted: 11 October 2017; Published: 24 October 2017.

doi: $10.21037 / \operatorname{tgh} .2017 .10 .02$

View this article at: http://dx.doi.org/10.21037/tgh.2017.10.02

\section{Introduction}

Gastric cancer ranks as the fifth most prevalent form of cancer worldwide, and the third most common cause of cancer-related death (1). Surgical resection is the sole curative treatment, in connection with regional lymphadenectomy as a recommended part of radical gastrectomy $(2,3)$. Systematic lymph dissection is crucial in gastric cancer surgery to achieve oncological clearance and a cure.

In 1994, Kitano and colleagues first reported laparoscopy-assisted Billroth I gastrectomy (4). Since then, laparoscopic gastrectomy (LG) has been associated with earlier patient recovery compared with open surgery, and has gained increasing international acceptance (5-8). Japan Society of Endoscopic Surgery (JSES) biennial surveys confirm the increasing use of laparoscopic procedures for treatment of gastric cancer in Japan (9). Its thirteenth national survey indicates that of 31,264 patients treated at Japanese institutions in 2015, approximately 9,500 (30.3\%) underwent LG, and laparoscopic distal gastrectomy (LDG) was the procedure most commonly performed.

Despite evidence supporting the efficacy of LDG for gastric cancer in the short term, however, uncertainty 


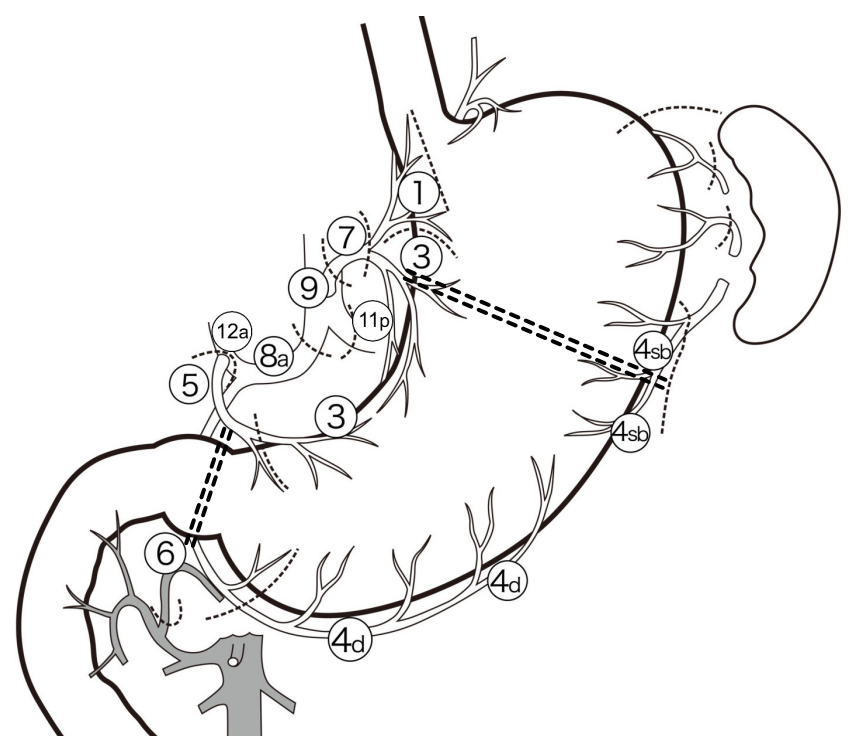

Figure 1 Summary of D2 lymph node dissection targets during distal gastrectomy.

remains concerning the efficacy of LG (10-14). Today, phase III randomized control trials on this procedure are ongoing in East Asian countries.

Distal gastrectomy (DG) is the most commonly performed mode of resection, and as appropriate surgical techniques need to be acquired by gastric surgeons, here we describe a 'gold standard' method to perform total LDG for advanced gastric cancers with curative intent.

\section{Methods}

\section{Surgical strategy}

The type of gastric resection is determined according to the following, based on Japanese treatment guidelines $(15,16)$ : the location and size of the tumor; the depth of invasion; and the nodal status of the tumor. DG is generally indicated for cancers in the distal and middle third part of the stomach, in which there are tumor margins of at least $2 \mathrm{~cm}$ for early and 3-5 cm for advanced lesions. Some patients with a very early disease can receive limited resection such as pylorus-preserving gastrectomy (17). This is indicated for lesions of the body of the stomach, but limited to the mucosa $(<4 \mathrm{~cm}$ in diameter) or submucosa $(<2 \mathrm{~cm}$ in diameter) where lymph node metastasis is not evident. For these procedures, a sufficient distal remnant is necessary to permit an antral cuff of at least $4 \mathrm{~cm}$ for gastrogastric anastomosis. A proximal gastrectomy procedure is indicated for patients with early gastric cancers in the upper third of the stomach with no evidence of lymph node metastasis. Total gastrectomy is indicated for locally advanced proximal tumors, or for multiple lesions for which the distal stomach cannot be preserved. Finally, local resection of the stomach is indicated for submucosal tumors of suspected malignancy.

Lymph node dissection is performed during vascular mobilization according to guidelines taking out lymph nodes en bloc on the side of the resection. Type D1 + lymph node dissection (stations 8a and 9 for DG) is performed for cancer thought to be extending submucosally, and type D2 (stations $8 \mathrm{a}, 9,11 \mathrm{p}$, and 12a for DG) is performed for all patients with tumors invading the muscle or with evidence of radiological or macroscopic lymph node involvement (16) (Figure 1).

The reconstruction method after DG is as follows: a Billroth I reconstruction by the delta-shaped method is normally performed (18-21), but Roux-en-Y reconstruction, which consists of totally mechanical gastrojejunostomy and an intracorporeal side-to-side jejunojejunostomy, is applied for patients with a small remnant stomach or hiatal hernia of the stomach (21-23) (Figure 2).

Indications for LG at our institute include the majority of tumors with the exception of diseases with a poor prognosis-such as bulky tumors, tumors invading the adjacent organs, and type 4 disease. To date, we prefer the open approach because of the oncological uncertainty of the laparoscopic procedure for these tumors.

\section{Surgical techniques}

\section{Overview of LDG for cancer}

As the most commonly performed laparoscopic procedure, LDG represents the basis from which all techniques in LG have developed.

* An incision is made in the gastrocolic ligament (proximal part of the greater omentum) just outside the gastroepiploic arcade on the body of the stomach.

* The gastrocolic ligament is divided parallel to and a few centimeters outside of the gastroepiploic arcade toward the left gastroepiploic artery (LGEA) in a superior direction and toward the left.

* The LGEA is identified, dissected, clipped, and divided using laparoscopic coagulating shears (LCS) or a sealing device (No. 4sb node dissection).

* The gastrocolic ligament is divided further proximally until an avascular plane is reached on the greater curvature beyond the level of gastric division. The 

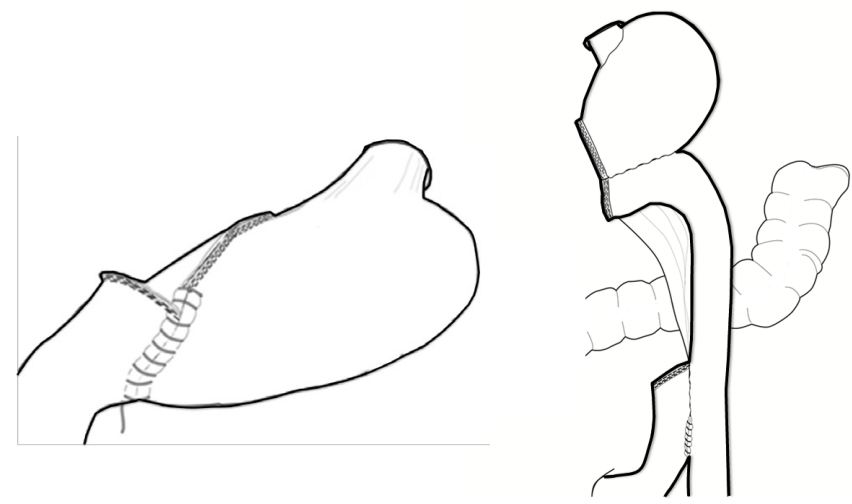

Figure 2 Diagram showing the protocol for intracorporeal stapled Billroth I and Roux-en-Y reconstructions.

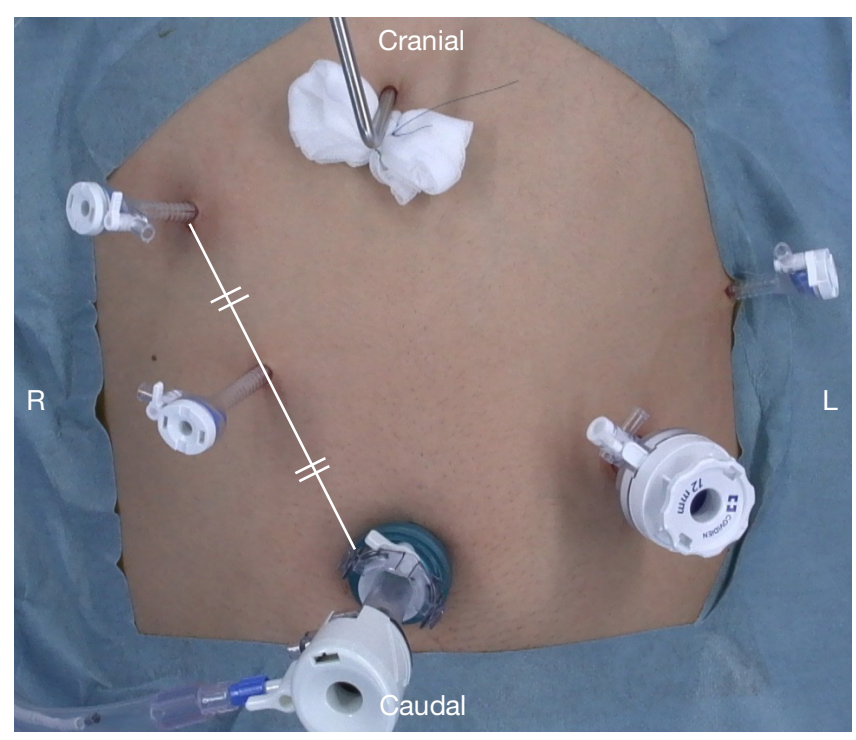

Figure 3 Port placement on the abdomen, and the Nathanson liver retractor.

perigastric fat is then stripped off the proximal region distally toward and beyond the level of gastric division and dissected en bloc with the main resection.

* The gastrocolic ligament is divided along the same plane distally toward the right side of the patient and the right gastroepiploic vessels. The No. $4 \mathrm{~d}$ lymph nodes are dissected in the same process.

* The right gastroepiploic vein (RGEV) and artery are dissected, clipped, and divided using LCS or the sealing device while dissecting No. 6 nodes in the process.

* The duodenum is retracted down to apply tension on the hepatoduodenal ligament, which is then incised to create a window to the space behind the duodenum.

* The neck (first part) of the duodenum is mobilized by clearing tissues off its wall toward the pylorus.

* A linear stapler is inserted to divide the duodenum just distal to the pylorus.

* The right gastric vein and artery are identified simultaneously and divided at the base, taking out No. 5 lymph nodes on the side of the resection. The left border of the portal vein can be followed for a No. 12a lymph node dissection.

* The lesser omentum is divided laterally close to the liver toward the abdominal esophagus.

* The peritoneum overlying the anterior surface of the pancreas is incised and the dissection is progressed cranially until the anterior surface of the common hepatic artery (CHA) is reached. This plane is followed over the artery to dissect the No. 8a lymph nodes. In about half of all cases, the left gastric vein (LGV) is found looping anterior to the CHA (posteriorly in other cases) and must be divided at this stage. Once over the artery, dissection of the deeper tissues behind the artery leads to the No. $8 \mathrm{p}$ lymph nodes.

* The left gastric pedicle is grasped close to the stomach and retracted ventrally to facilitate dissection around the root of the left gastric artery (LGA). This is divided taking out the No. 7 lymph nodes in the process.

* The dissection is continued posteriorly along the anterior surface of the celiac trunk toward the diaphragmatic hiatus for the No. 9 lymph nodes. Extension of this dissection laterally along the splenic artery allows for dissection of No. 11p lymph nodes.

* Lesser curvature lymph nodes (No. 3 and No. 1 proximally) are separated from the gastric wall of the stomach.

* Once the gastric wall at the level division is denuded of its perigastric fat circumferentially, the stomach is transected with the linear stapler.

* A specimen pouch is used to retrieve the specimen with its attached lymph nodes.

* Reconstruction is commenced.

\section{Port placement and liver retraction (Figure 3)}

The patient is positioned with his or her hips extended and abducted. A pneumoperitoneum (12 $\mathrm{mmHg})$ is established through an umbilical trocar, and the umbilical optical port serves as a reference point for the insertion of ports on 


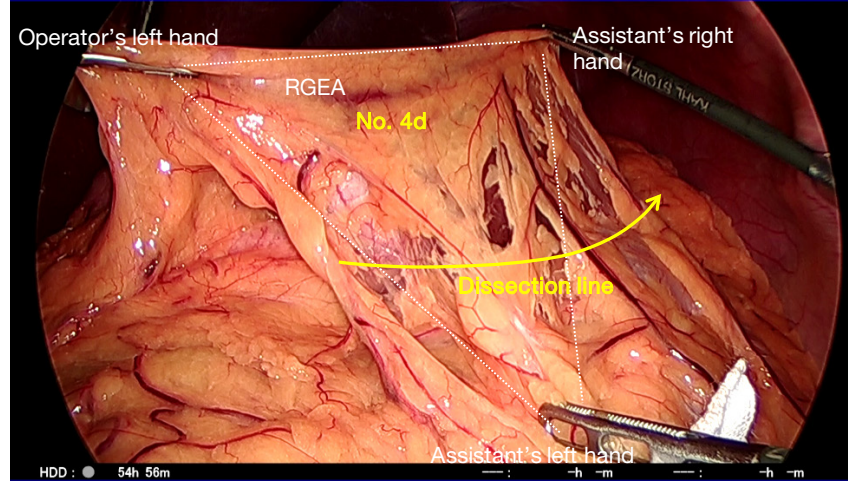

Figure 4 Division of the gastrocolic ligament. RGEA, right gastroepiploic artery.

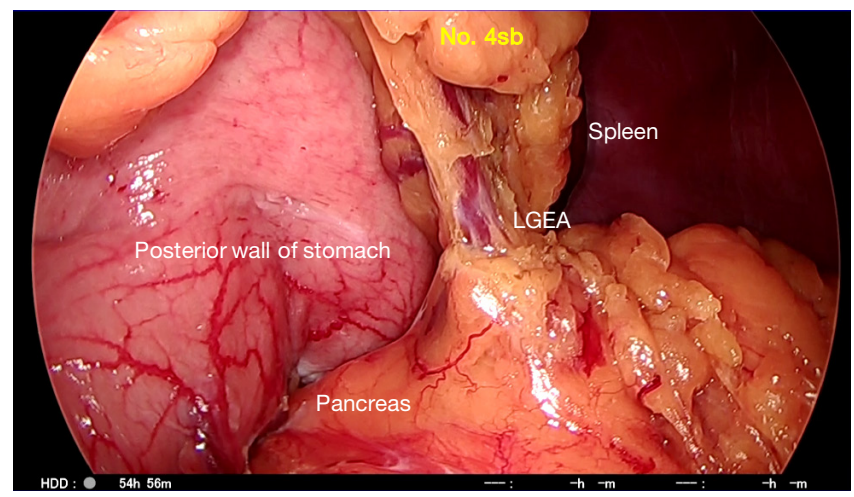

Figure 5 Division of the left gastroepiploic artery. LGEA, left gastroepiploic artery.

either side of the patient's abdomen. Liver retraction is achieved using a Nathanson liver retractor and a Prolene thread (19,24).

Division of the gastrocolic ligament to the left (Figure 4) The primary surgeon standing on the patient's right side grasps the greater curvature close to the right gastroepiploic vessels, and the assistant, standing on the patient's left, grasps the gastrocolic ligament at the same level on the side of the transverse colon with their left hand, thus creating a clear operative plane. The assistant applies tension on the fat to help the operator divide the gastrocolic ligament to enter the lesser sac (the bursa omentalis). While maintaining a distance of 3-4 cm away from the gastroepiploic arcade, the division of the gastrocolic ligament is continued proximally toward the patient's left. Tissue division is usually performed using LCS or a sealing device. Together with the left-hand grasper of the operator and using both hands of the assistant, a triangular retraction of tissues is desirable. The left hand of the operator retracts the greater curve in a 10 o'clock direction on the monitor. The left hand of the assistant on the left retracts the greater omentum in a 4 o'clock direction and is used to apply the appropriate amount of tension on the tissues that are to be divided. Care must be taken not to damage the transverse colon. The assistant grasps the tissues close to the colon while protecting the bowel wall with the grasper as the dissection is continued toward the lower pole of the spleen where structures are much closer to each other.

\section{Division of the left gastroepiploic vessels and dissection} of No. 4sb lymph nodes (Figure 5)

While approaching the left gastroepiploic pedicle, the operator's left hand is used to grasp the posterior wall of the stomach near the greater curvature, and then lift it in a 10 o'clock direction to apply tension on the pedicle and gastrosplenic ligament. This helps to make space and clarify the anatomy, facilitating dissection of the left gastroepiploic vessels. Care must be taken to avoid excessive tension that may lead to traction injury to the spleen. Adhesions to the posterior gastric wall or the gastrocolic ligament are common, and need to be divided using diathermy to improve exposure. The left gastroepiploic vessels are identified medially rising from the retroperitoneum toward the greater wall of the stomach. The peritoneal layer is incised and the vessels are dissected using an energy device. The vessels are clipped at the base and divided above the clips, taking out the No. 4sb lymph nodes at the same time. The space beneath the gastrosplenic ligament is entered to identify an avascular plane on the greater curvature of the gastric wall. Tissues are then divided to allow the operator to reach the gastric wall and denude it of its perigastric fat.

Using diathermy, a window is created in the perigastric fat distal to the line of gastric division. An energy device is used to separate the perigastric lymphovascular tissues from the gastric wall proximally and reach the already performed lateral dissection. This denudes the proximal greater curvature of the stomach in preparation for gastric division with the linear stapler. The lymph nodes are separated from the gastric wall that will be left behind, but remain connected to the main resection. This part of the procedure is sometimes performed later, immediately before the stomach is divided.

\section{Dissection of No. 4d lymph nodes (Figure 6)}

The primary surgeon and the assistant swap sides (the 


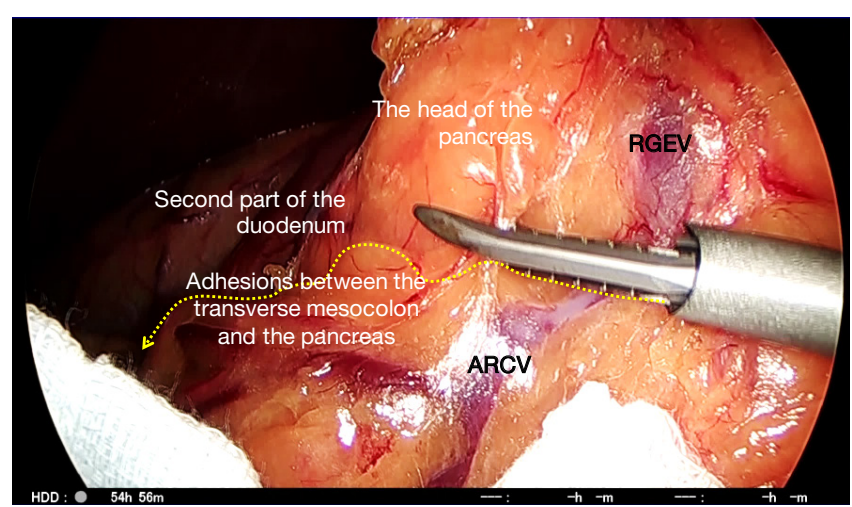

Figure 6 Route for dropping down the transverse mesocolon from the head of the pancreas. RGEV, right gastroepiploic vein; ARCV, accessory right colic vein.

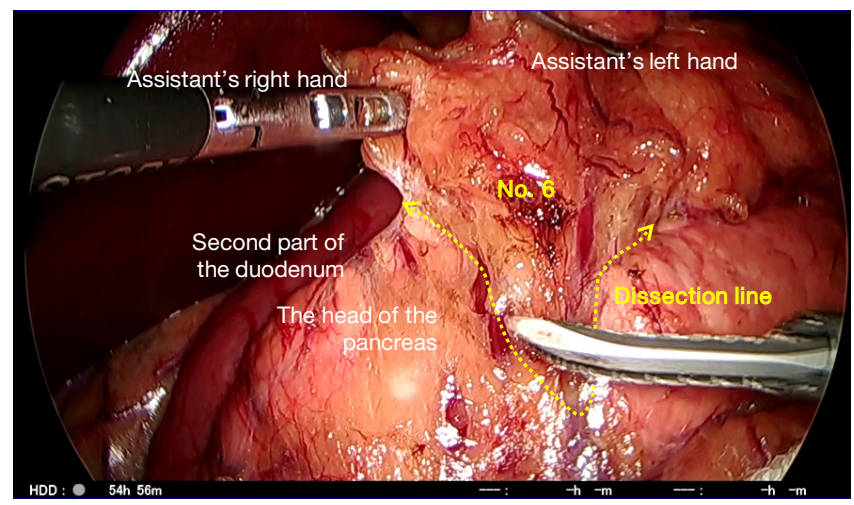

Figure 7 Dissection of the No. 6 lymph nodes.

operator to the patient's left, and the assistant to the right). Division of the gastrocolic ligament along the same line parallel to the gastroepiploic arcade continues distally toward the patient's right. The assistant (now on the right) holds the greater curvature of the stomach using both graspers and lifts it in a 12 o'clock direction to create a space behind the stomach and thereby apply tension to the undivided distal gastrocolic ligament. Adhesions to the anterior surface of the pancreas are then divided to enable further lifting of the stomach and dissection toward the duodenum. The plane between the transverse mesocolon and the greater omentum is followed while dividing the gastrocolic ligament, and any adhesions crossing this region should be divided. The peritoneal reflection at the right lower limit of the lesser sac is then reached, and beyond this, the omental fat is separated by staying in a plane anterior to the transverse mesocolon. The assistant must adjust the ventral retraction of the stomach frequently and apply the correct amount of tension for each step of the dissection. As the transverse mesocolon tents upwards, it must be separated from the omentum with a combination of blunt dissection with graspers and sharp dissection with the energy device to allow it to drop. Finally, the inner curve of the C-loop on the second part of the duodenum is reached with the right gastroepiploic pedicle still intact (Figure 6). The membrane between the duodenum and the pancreas is divided back toward the pylorus, which defines the lateral limit of the No. 6 dissection (below).

Division of the right gastroepiploic vessels and dissection of No. 6 lymph nodes (Figures 6 and 7)

The position of the pylorus is determined on its anterior surface, and dissection commences over the anterior surface of the pancreas toward the right gastroepiploic vessels. The assistant's left grasper is used to lift the perigastric fat surrounding the right gastroepiploic pedicle near the pylorus to apply tension on the tissues. The origin of the right gastroepiploic artery is typically found $2 \mathrm{~cm}$ below the pylorus in this configuration. The head and the inferior border of the body of the pancreas are used for orientation during this part of the procedure. The plane anterior to the transverse mesocolon is followed over the pancreas, and the accessory right colic vein (ARCV,) and gastrocolic trunk might be visible within the mesenteric fat (Figure 6). Laterally, the anterior superior pancreaticoduodenal vein might be identified and this can be followed to its drainage to the RGEV. Once dissected, the operator clips the RGEV above the anterior superior pancreaticoduodenal tributary and a sealing device or LCS is used to seal and divide above this clip (Figure 7). The anterior surface of the pancreas is further exposed cranially on either side of the right gastroepiploic artery. Care must be taken to avoid damage to the pancreas while its head is tented up by traction. Energy devices can cause thermal injury here as well as during dissection around the common hepatic and splenic arteries. This can lead to postoperative pancreatitis. The gastroduodenal artery (GDA) may become visible behind the duodenum and this can be followed to the origin of the right gastroepiploic artery. The artery is dissected, clipped at the base, sealed, and divided above the clip using a coagulation device. The infrapyloric artery passing to the pylorus can often be seen and this might also need to be clipped or sealed. Once more space has been created posterior to the duodenum, the remaining tissues are divided to mobilize the duodenum further. The perigastric 


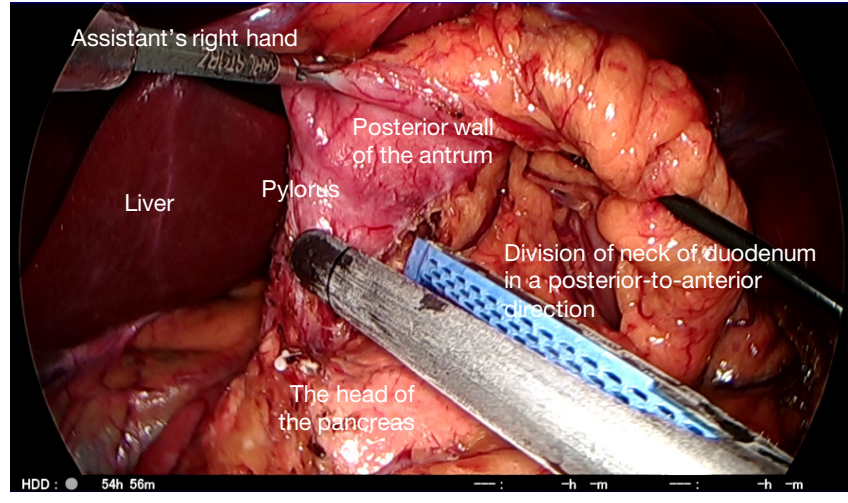

Figure 8 Division of the neck of the duodenum in a posterior-toanterior direction (for the Billroth I operation).

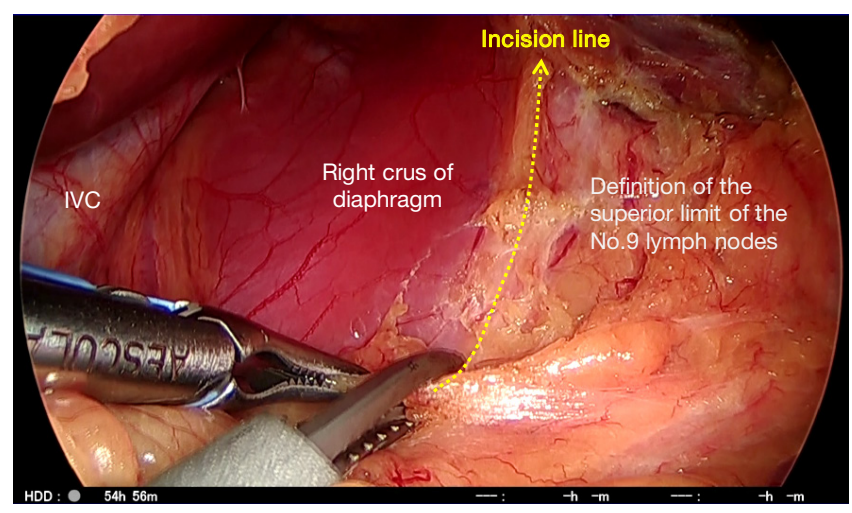

Figure 9 Division of the peritoneum along the right crus of the diaphragm to define the superior limit of the No. 9 lymph nodes. IVC, inferior vena cava.

tissues are divided toward the pylorus while denuding the duodenal wall posteriorly. The GDA can be followed toward its branching from the hepatic artery, which defines the upper limit of the dissection from this approach. Several arterial branches to the lesser curve of the duodenum can also be identified and divided from this viewpoint.

\section{Division of the duodenum (Figure 8)}

A sterile gauze pad is packed behind the pylorus to help with mobilization of the lesser curvature of the stomach. The hepatoduodenal ligament is divided above the duodenal cap, by incising the peritoneal layer using monopolar diathermy. In thin patients, the gauze might be visible through the transparent membrane and the incision can then be made in an avascular region. A wide window should be made by dividing the vascular tissues along the lesser curvature of the stomach using bipolar diathermy or a coagulation device. Once the duodenal neck has been mobilized completely from above and below, the linear stapler is inserted to divide the duodenum distal to the pylorus.

The assistant on the right side of the patient rotates the duodenum by pulling the posterior wall of the gastric antrum from below, and by rotating the stomach upward. This is done to rotate the duodenum so that it can be divided in a posterior-to-anterior direction rather than along the greater to the lesser curvatures. This enables subsequent gastroduodenostomy by maintaining an adequate blood supply to the anastomosis line $(17,18,20,25)$.

\section{Division of the lesser omentum (Figure 9)}

The primary surgeon and the assistant again swap sides (the operator is now on the patient's right side, and the assistant is on the left). The hepatogastric ligament, forming the proximal membranous part of the lesser omentum, is divided toward the abdominal esophagus. A coagulation device is used to divide the fatty layers while membranes are divided using monopolar diathermy. In $15-20 \%$ of patients, an accessory left hepatic artery runs from the lesser curvature of the stomach to the liver. This needs to be dissected, and its size and any contribution to the hepatic circulation evaluated. Once the upper limit near the hiatus is reached, the peritoneum overlying the right crus of the diaphragm must be divided to define the superior and right lateral limits of dissection of the No. 9 lymph nodes located cranial to the celiac trunk (Figure 9).

\section{Dissection of No. 5 lymph nodes (Figures 10 and 11)}

The incision of the hepatoduodenal ligament is continued upwards toward the previous dissection to expose the lateral border of the hepatic artery using sharp dissection. There are large nerve fibers around the hepatic artery in this area, and these must be distinguished from any lymphatics and small blood vessels. Inferiorly, the plane on the anterior surface of the pancreas is followed from below to expose the anterior nerve plexus around the CHA and No. 8a lymph nodes (Figure 10). The assistant on the left side grasps the right gastric artery (RGA) in a $2-3$ o'clock direction to apply traction, which facilitates dissection around its origin (Figure 11). This can occasionally tent up the hepatic artery, so care must be taken not to divide this. The RGA might also arise from the left hepatic artery. There are frequent anatomical variations in this region, and hepatic ischemia must be avoided (26). This dissection plane can be followed laterally and superiorly to separate the No. 12a lymph nodes 


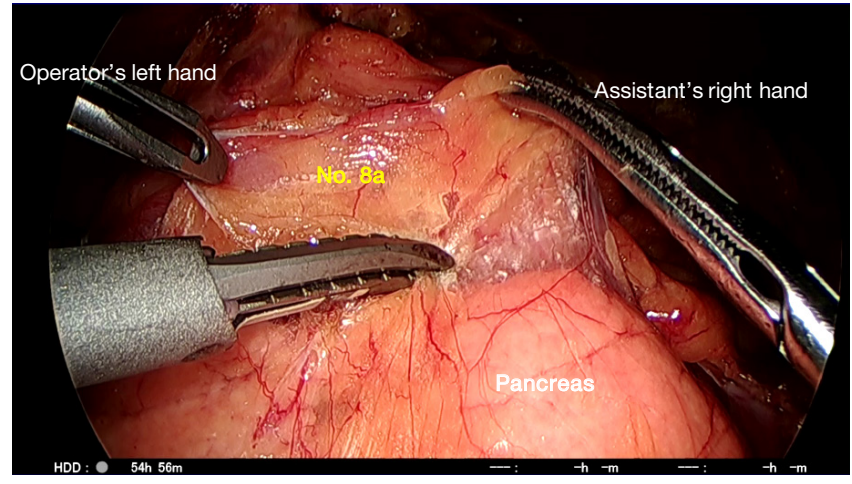

Figure 10 Incision of the peritoneum along the superior border of the pancreas and removal of No. 8a lymph nodes.

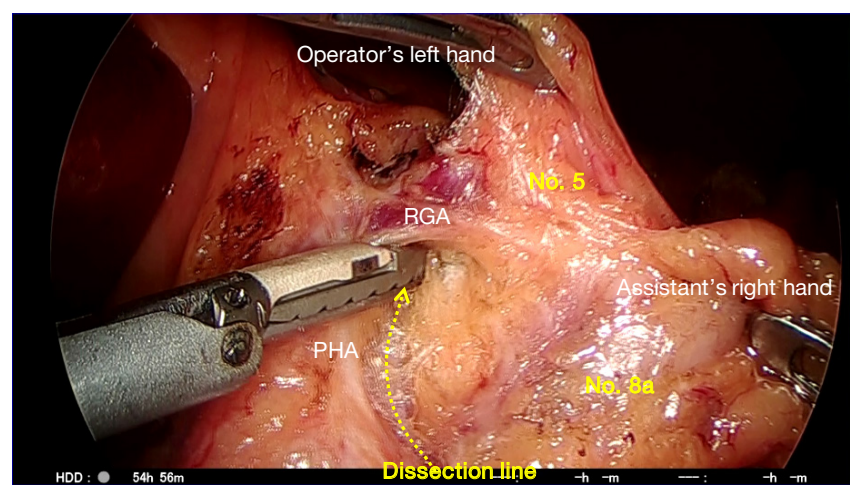

Figure 11 Access to the route of the right gastric artery (RGA). PHA, proper hepatic artery.

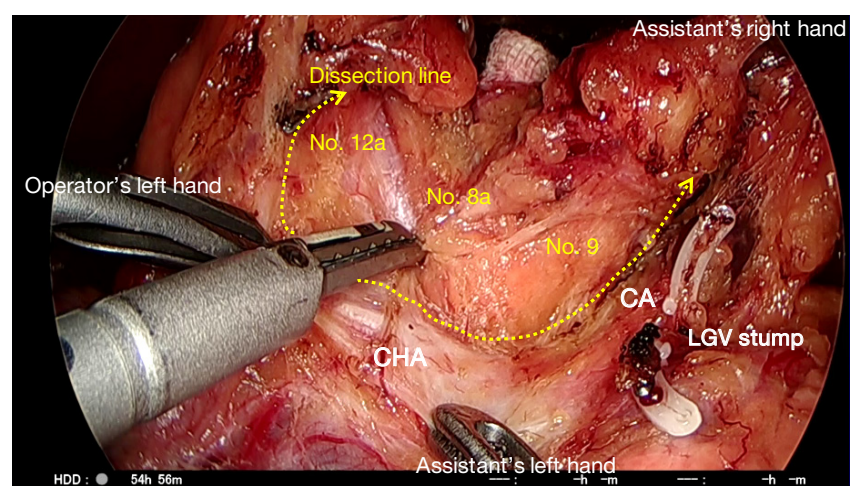

Figure 12 En bloc dissection of No. 8a, 12a, and 9 lymph nodes along the outermost layer. CA, celiac axis; CHA, common hepatic artery; LGV, left gastric vein.

from the hepatic artery proper. Dividing the origin of the RGA completes the dissection of the No. 5 lymph nodes.

\section{Dissection of No. 8a lymph nodes (Figure 12)}

The previous dissection is continued exposing the anterior nerve plexus around the CHA, medially toward the LGA. The operator must grasp the lymph nodes gently with a grasper in the left hand and continue to separate the plane between the artery and lymph nodes using monopolar diathermy or the LCS. In a prophylactic lymphadenectomy, en bloc dissection of the No. 8a, 12a and 9 lymph nodes should be carried out along the outermost layer of the CHA (Figure 12). The outermost layer refers to the dissectible layer around the artery (27). The anterior surface of the pancreas is rolled away by the assistant, and this should facilitate exposure for deeper dissection. Small blood vessels passing from the pancreas to the lymph nodes should be precoagulated using soft coagulation monopolar diathermy or bipolar forceps before sharp division using cutting mode diathermy. Following the outermost layer of the CHA allows for dissection of the No. 8a lymph nodes. Continuing posteriorly beyond the artery, the lymphatic tissue can be divided straight across and above the No. 8 p nodes or deeper beneath these nodes before reaching the plane for No. 9 dissection. Devices such as the LigaSure ${ }^{\mathrm{TM}}$ and LCS can be used for simultaneous sealing and dividing the tissues. The LGV might be encountered toward the pedicle in about half of patients, where it drains directly into the portal vein or into its junction with the splenic vein behind the CHA. In most other patients, the vein loops anterior to the artery to drain into the splenic vein (26). Rarely, the vein might run posterior to the splenic artery, which drains into the splenic vein. Once identified, the LGV should be clipped and divided.

\section{Dissection of No. 9, 7, and 11p lymph nodes (Figures 13 and 14)}

Using a grasper in the right hand, the assistant grasps the left gastric pedicle close to the stomach and lifts it ventrally. The peritoneal incision along the superior border of the pancreas is extended as far to the patient's left as possible to expose the surfaces of the CHA and the splenic artery in the same plane. The tissues are dissected with the operator's right hand using dissecting forceps and devices such as LCS.

The dissection is continued medially toward the LGA. The plane over the common hepatic and splenic artery is followed to reach the origin of the LGA. No. 7 lymph nodes are dissected off the surface of the artery and the vessel is also cleared of its surrounding vagal branches. 


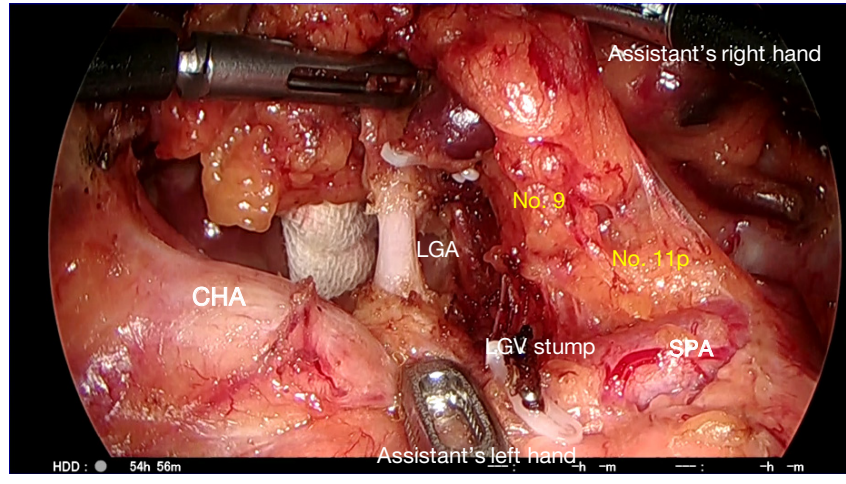

Figure 13 Division of the left gastric artery (LGA). CHA, common hepatic artery; LGV, left gastric vein; SPA, splenic artery.

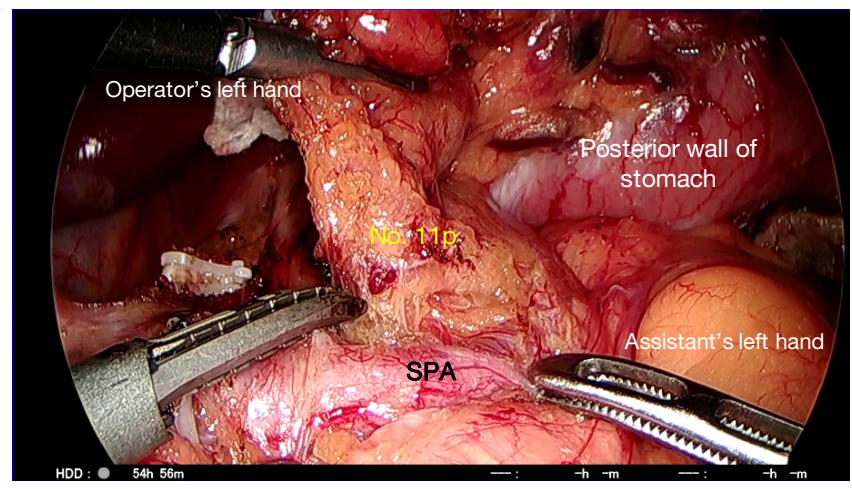

Figure 14 No. 11p lymph node dissection around the splenic artery (SPA).

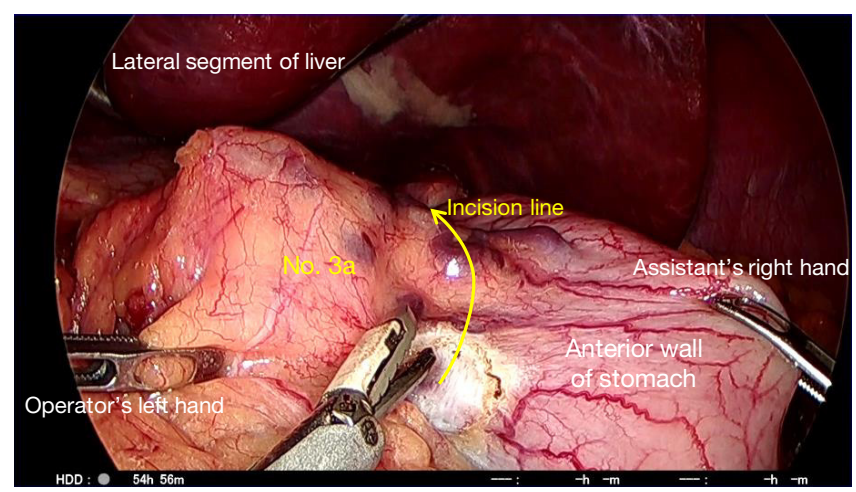

Figure 15 Stripping of the lesser omentum (No. 3a lymph nodes) from the lesser curvature of the stomach.

Once the origin is identified and the vessel is skeletonized, it is clipped and then divided above the clip (Figure 13). The dissection is then continued onto the left crus of the diaphragm to define the left margin of the No. 9 lymph nodes. This plane can then be connected to the already dissected plane on the right crus to define the posterior limit of No. 9 node dissection.

In cases where there is an accessory left hepatic artery that needs to be preserved, the dissection is continued along the LGA to clear the vessel away from its origin. Once the branching of the accessory left hepatic artery has been identified, two or three small branches to the lesser curvature of the stomach are clipped and divided, taking the lymphatic tissue on the side of the stomach and preserving the accessory left hepatic artery (26).

The loose connective tissue to the left of the left gastric pedicle can be dissected to complete the dissection of No. $11 \mathrm{p}$ lymph nodes (Figure 14). This is a fascial plane formed by fusion of visceral with parietal peritoneum. Cranial-tocaudal mobilization of the distal pancreas along the fusion fascia should facilitate adequate oncological clearance around the splenic artery. The dissection of No. 11p nodes is followed until the posterior gastric artery has been reached to the far left, and the splenic vein might become visible as the posterior border in some patients during this dissection.

Dissection of No. 1 and No. 3 lymph nodes (Figure 15) The stomach is returned to its natural position and the position of the preoperatively placed marking tattoo of India ink is confirmed (see below). The left hand of the operator is used to retract the fat of lesser omentum while the assistant retracts the gastric wall near the lesser curve of the proximal stomach to apply tension on the tissues. The peritoneal layer just distal to the line of gastric division is incised using monopolar diathermy and an atraumatic grasper is inserted proximally to create a space between the anterior and posterior laminae of the lesser omentum. The anterior leaf is separated from the gastric wall using a LigaSure ${ }^{\mathrm{TM}}$ or LCS and an atraumatic grasper is used to dissect it further proximally. Retraction of the stomach in a caudal direction by the assistant facilitates progressive proximal dissection. Once the anterior leaf is completely free, the same technique is used for the posterior leaf, going from distal to proximal. This is performed until the gastric wall of the proximal lesser curvature is bare and stripped of its perigastric tissues.

Division of the stomach and specimen retrieval (Figure 16) Gastric resection lines must be determined accurately 


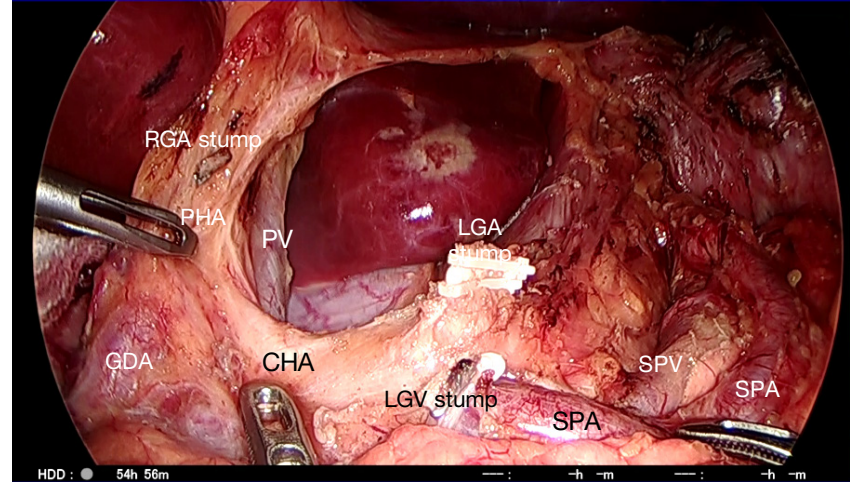

Figure 16 Field of view at the completion of D2 lymph node removal and distal gastrectomy. RGA, right gastric artery; PHA, proper hepatic artery; PV, portal vein; GDA; gastroduodenal artery; CHA, common hepatic artery; LGA, left gastric artery; LGV, left gastric vein; SPA, splenic artery; SPV, splenic vein.

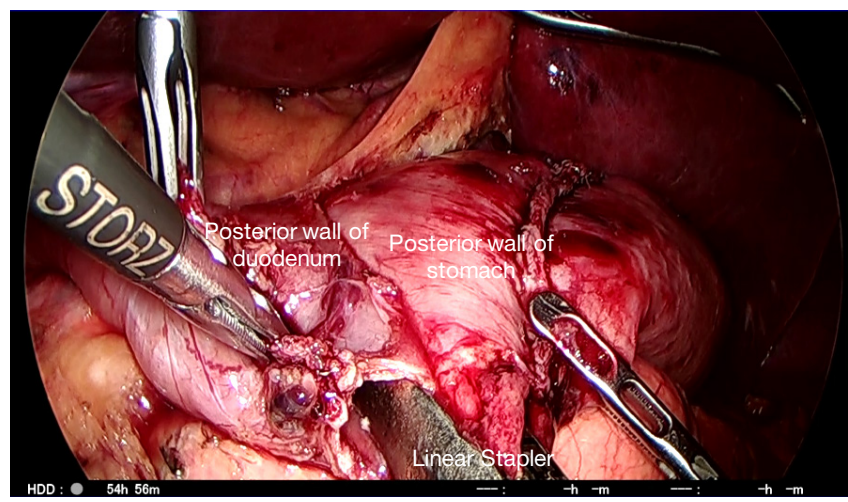

Figure 17 Each fork of the stapler is positioned to join the posterior walls of the stomach and duodenum.

relative to the tattoo mark of the lesion. The tattoo of India ink must be injected into the submucosal space just proximal to the tumor during preoperative endoscopy. If tattooing or macroscopic appearance are unreliable, endoscopic clipping and intraoperative fluoroscopy can be used to determine the level of gastric division.

The retrieval bag is inserted through the $12-\mathrm{mm}$ port and opened in the upper abdomen. After the operator has placed the resection specimen together with attached lymph nodes inside, the bag is closed and retrieved from the abdominal cavity through the umbilical wound, which is extended superiorly to $4-5 \mathrm{~cm}$. This is then closed leaving

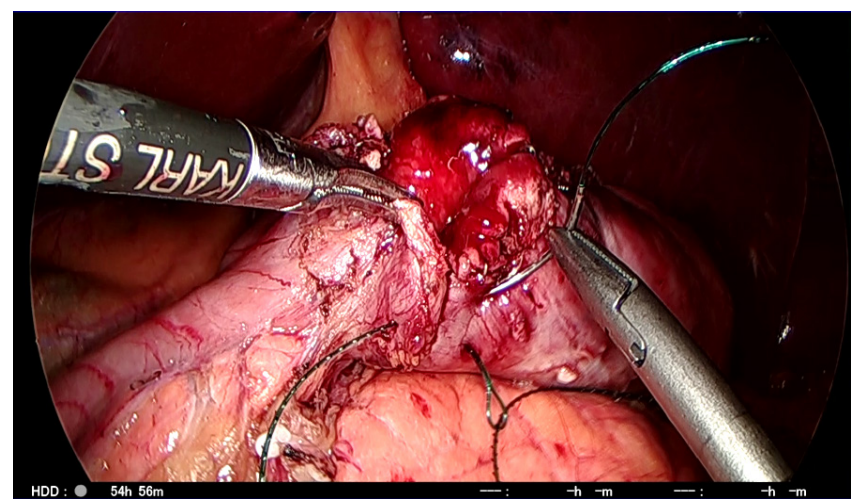

Figure 18 Step 1 of the intracorporeal suture closure of the common enterotomy hole used for the stapler. This shows fullthickness inner layer closure using a knotless unidirectional barbed suture, starting from the corner of the greater curvature of the stomach.

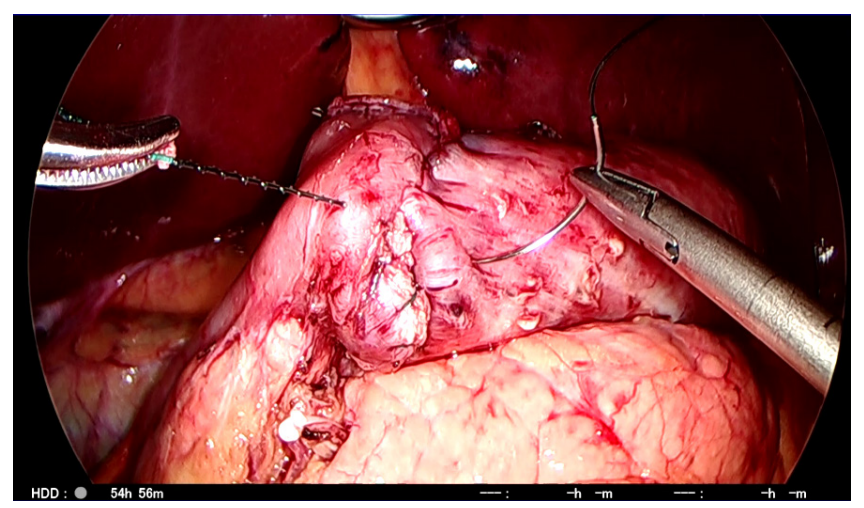

Figure 19 Step 2 of the intracorporeal suture closure of the common enterotomy hole used for the stapler. This shows closure of the second seromuscular layer using the same suture, returning from the lesser curvature of the stomach.

a gap for reinsertion of the optical port. The gas tube and camera are transferred back to the reinserted umbilical port and a pneumoperitoneum is reestablished.

\section{Billroth I gastroduodenostomy (Figures 17-19)}

Billroth I gastroduodenostomy is the first choice following DG as it is the most anatomical reconstruction. The Rouxen-Y approach is reserved for when there is too much tension for a primary gastroduodenal anastomosis. Rouxen- $\mathrm{Y}$ is also indicated if the patient suffers from reflux esophagitis, has a hiatus hernia or is elderly or high risk $(19,22,28)$. 
A gastrotomy is made on the greater curvature corner of the staple line on the remnant stomach followed by a similar hole on the inferior staple line corner of the transected duodenum. The cartridge fork of the 45-mm long linear stapler is inserted into the gastric remnant through the $12-\mathrm{mm}$ port on the left. While the right hand of the assistant on the patient's left side pulls the staple line anteriorly, the stapler is closed temporarily ensuring that the posterior gastric wall is held between the jaws. The stapler with the stomach is moved slowly toward the duodenum. While the assistant pulls on the gastric staple line in a 3 o'clock direction, the stapler is opened to insert the anvil fork into the duodenum. This is best performed by the operator on the right side of the patient using the two graspers, who gently feeds the duodenum over the anvil fork of the stapler. The surgeons together pull on the staple lines to ensure that the two posterior walls meet. When the enterotomy edges are in line, the stapler is fired to create the end-to-end anastomosis (Figure 17) $(18,20)$.

After checking for any bleeding in the $\mathrm{V}$-shaped anastomotic line on the posterior wall, the opening hole for a linear stapler is closed using an Albert-Lembert 2-layer suture with a knotless unidirectional barbed suture for both layers. A full-thickness inner layer closure commences from the corner of the greater curvature toward the lesser curvature using a continuous suturing technique (Figure 18). Once the full-thickness layer has been completed, suturing of the second seromuscular layer returns toward the greater curvature using the same barbed suture (Figure 19). After the last stitch, the suture is cut without any knots to anchor the last cast. This completes the intra-abdominal Billroth I anastomosis $(20,21)$.

\section{Results}

From April 2000 to December 2016, 1,350 patients with gastric cancer underwent laparoscopic resections with curative intent at Osaka Medical College Hospital, Japan. We performed DG in 787 patients, pylorus-preserving gastrectomy in 243 , proximal gastrectomy in 123 , total gastrectomy in 111 , and wedge resection in 86 . The grade 3 or higher morbidity rate was $6.1 \%$, and the postoperative mortality rate was $0.3 \%$. The 5 -year overall and disease-free survival rates were $91 \%$ and $85 \%$, respectively. According to tumor stage, the 5-year overall and disease-free patient survival rates were $95.6 \%$ and $92.4 \%$ for stage IA disease, $88.6 \%$ and $83.9 \%$ for stage IB disease, $82.4 \%$ and $71.9 \%$ for stage IIA disease, and $72.2 \%$ and $66.3 \%$ for stage IIB disease, respectively.

\section{Discussion}

The LG procedure was introduced in our unit in 2000 to treat patients with early gastric cancers. Based on our clinical experience and on improvements in our technique, the indications have been expanded to include those with advanced gastric cancers $(19,21,26)$. In 2010 in a singlecenter retrospective study of $601 \mathrm{LG}$ procedures, we reported overall 5 -year patient survival rates of $93.4 \%$ for those with T1 tumors, $70.0 \%$ for those with T2 tumors, and $57.1 \%$ for those with $\mathrm{T} 3$ tumors. These were similar to the results of open resection $(29,30)$.

Although originally we began performing laparoscopyassisted DG to overcome the drawbacks of cumbersome reconstructions, in 2004 we introduced intracorporeal stapled gastroduodenostomy and gastrojejunostomy along with LDG $(18,22)$. Through our experience, we have noted that intracorporeal anastomosis subsequent to LDG has several advantages over laparoscopy-assisted LG with extracorporeal anastomosis, including a small wound, reduced invasiveness, and a safe anastomosis (28). However, intracorporeal suturing and knot tying for anastomoses remain tedious and time-consuming procedures in such surgery. The surgery requires safe and reproducible techniques due to leaks an fistulas resulting in morbidity and mortality. We hypothesized that a barbed suture might improve the efficiency and reduce the cost of intracorporeal reconstruction of the digestive tract, by closing the entry hole of the stapler instead of stapling, and by minimizing the time required to suture. Therefore, in 2011 we changed to using barbed sutures to close the entry hole for the linear stapler. By the end of 2016, we had performed 571 intracorporeal anastomoses with the use of barbed sutures in 354 patients with gastric cancer. Encouragingly, only one patient developed an anastomotic complication related to postoperative bleeding. There was no need to convert to usual sutures or mechanical closure of the entry hole, or for reoperations because of adhesive obstructions. Along with reduced mortality, this was seen over a median followup duration of 30.9 months. Through our experience, we strongly believe that this novel technique can achieve better surgical outcomes in patients requiring LG. Since its first use for gastric cancer in 1991, minimally invasive surgery for gastric cancer has received general acceptance. Widespread acceptance of LG has resulted from improvements in equipment and laparoscopic techniques, 
not only for DG but for other types of gastric resection. Currently, in the hope of minimizing the invasiveness of the procedure and improving access, laparoscopic surgery has been used in older patients and in those with cancer of the gastroesophageal junction.

Large-scale trials have confirmed the safety of LG internationally, and large-scale multicenter clinical trials have been launched to establish oncologic evidence in East Asia (10-14). We consider that conclusive evidence for the safe use of LG for patients with gastric cancer will emerge from this region. Following these developments, global knowledge sharing and cooperation on the surgical treatment of gastric cancer will be essential.

\section{Acknowledgements}

None.

\section{Footnote}

Conflicts of Interest: The authors have no conflicts of interest to declare.

\section{References}

1. Ferlay J, Soerjomataram I, Dikshit R, et al. Cancer incidence and mortality worldwide: sources, methods and major patterns in GLOBOCAN 2012. Int J Cancer 2015;136:E359-86.

2. Sano T, Sasako M, Yamamoto S, et al. Gastric cancer surgery: morbidity and mortality results from a prospective randomized controlled trial comparing D2 and extended para-aortic lymphadenectomy--Japan Clinical Oncology Group study 9501. J Clin Oncol 2004;22:2767-73.

3. Songun I, Putter H, Kranenbarg EM, et al. Surgical treatment of gastric cancer: 15-year follow-up results of the randomised nationwide Dutch D1D2 trial. Lancet Oncol 2010;11:439-49.

4. Kitano S, Iso Y, Moriyama M, et al. Laparoscopyassisted Billroth I gastrectomy. Surg Laparosc Endosc 1994;4:146-8.

5. Kitano S, Shiraishi N, Fujii K, et al. A randomized controlled trial comparing open vs laparoscopy-assisted distal gastrectomy for the treatment of early gastric cancer: an interim report. Surgery 2002;131:S306-11.

6. Huscher CG, Mingoli A, Sgarzini G, et al. Laparoscopic versus open subtotal gastrectomy for distal gastric cancer: five-year results of a randomized prospective trial. Ann
Surg 2005;241:232-7.

7. Adachi Y, Shiraishi N, Shiromizu A, et al. Laparoscopyassisted Billroth I gastrectomy compared with conventional open gastrectomy. Arch Surg 2000;135:806-10.

8. Liakakos T, Roukos DH. Randomized evidence for laparoscopic gastrectomy short-term quality of life improvement and challenges for improving long-term outcomes. Ann Surg 2009;250:349-50; author reply 50.

9. Etoh T, Shiraishi N, Kitano S. Current trends of laparoscopic gastrectomy for gastric cancer in Japan. Asian Journal of Endoscopic Surgery 2009;2:18-23.

10. Katai H, Sasako M, Fukuda H, et al. Safety and feasibility of laparoscopy-assisted distal gastrectomy with suprapancreatic nodal dissection for clinical stage I gastric cancer: a multicenter phase II trial (JCOG 0703). Gastric Cancer 2010;13:238-44.

11. Inaki N, Etoh T, Ohyama T, et al. A Multi-institutional, Prospective, Phase II Feasibility Study of LaparoscopyAssisted Distal Gastrectomy with D2 Lymph Node Dissection for Locally Advanced Gastric Cancer (JLSSG0901). World J Surg 2015;39:2734-41.

12. Kim W, Kim HH, Han SU, et al. Decreased Morbidity of Laparoscopic Distal Gastrectomy Compared With Open Distal Gastrectomy for Stage I Gastric Cancer: Short-term Outcomes From a Multicenter Randomized Controlled Trial (KLASS-01). Ann Surg 2016;263:28-35.

13. Kim HH, Hyung WJ, Cho GS, et al. Morbidity and mortality of laparoscopic gastrectomy versus open gastrectomy for gastric cancer: an interim report--a phase III multicenter, prospective, randomized Trial (KLASS Trial). Ann Surg 2010;251:417-20.

14. Yu J, Hu J, Huang C, et al. The impact of age and comorbidity on postoperative complications in patients with advanced gastric cancer after laparoscopic D2 gastrectomy: results from the Chinese laparoscropic gastrointestinal surgery study (CLASS) group. Eur J Surg Oncol 2013;39:1144-9.

15. Japanese classification of gastric carcinoma: 3rd English edition. Gastric Cancer 2011;14:101-12.

16. Japanese Gastric Cancer Association. Japanese gastric cancer treatment guidelines 2010 (ver. 3). Gastric Cancer 2011;14:113-23.

17. Lee SW, Bouras G, Nomura E, et al. Intracorporeal stapled anastomosis following laparoscopic segmental gastrectomy for gastric cancer: technical report and surgical outcomes. Surg Endosc 2010;24:1774-80.

18. Kanaya S, Gomi T, Momoi H, et al. Delta-shaped anastomosis in totally laparoscopic Billroth I gastrectomy: 
new technique of intraabdominal gastroduodenostomy. J Am Coll Surg 2002;195:284-7.

19. Lee SW, Nomura E, Bouras G, et al. Long-term oncologic outcomes from laparoscopic gastrectomy for gastric cancer: a single-center experience of 601 consecutive resections. J Am Coll Surg 2010;211:33-40.

20. Lee SW, Nomura E, Tokuhara T, et al. Laparoscopic technique and initial experience with knotless, unidirectional barbed suture closure for staple-conserving, delta-shaped gastroduodenostomy after distal gastrectomy. J Am Coll Surg 2011;213:e39-45.

21. Lee SW, Kawai M, Tashiro K, et al. Laparoscopic gastrointestinal anastomoses using knotless barbed absorbable sutures are safe and reproducible: a singlecenter experience with 242 patients. Jpn J Clin Oncol 2016;46:329-35.

22. Takaori K, Nomura E, Mabuchi H, et al. A secure technique of intracorporeal Roux-Y reconstruction after laparoscopic distal gastrectomy. Am J Surg 2005;189:178-83.

23. Laparoscopic gastrointestinal anastomoses using knotless barbed absorbable sutures are safe and reproducible: a single-center experience with 242 patients. Bouras G, Lee SW, Nomura E, et al. Surgical outcomes from laparoscopic distal gastrectomy and Roux-en-Y reconstruction: evolution in a totally intracorporeal technique. Surg

doi: $10.21037 / \operatorname{tgh} .2017 .10 .02$

Cite this article as: Lee SW, Kawai M, Tashiro K, Bouras G, Kawashima S, Tanaka R, Nomura E, Uchiyama K. Laparoscopic distal gastrectomy with D2 lymphadenectomy followed by intracorporeal gastroduodenostomy for advanced gastric cancer: technical guide and tips. Transl Gastroenterol Hepatol 2017;2:84.
Laparosc Endosc Percutan Tech 2011;21:37-41.

24. Bann S, Butler A, Shaul T, et al. A technique for insertion of the laparoscopic Nathanson liver retractor. Ann R Coll Surg Engl 2005;87:472-3.

25. Kanaya S, Kawamura Y, Kawada H, et al. The delta-shaped anastomosis in laparoscopic distal gastrectomy: analysis of the initial 100 consecutive procedures of intracorporeal gastroduodenostomy. Gastric Cancer 2011;14:365-71.

26. Lee SW, Shinohara H, Matsuki M, et al. Preoperative simulation of vascular anatomy by three-dimensional computed tomography imaging in laparoscopic gastric cancer surgery. J Am Coll Surg 2003;197:927-36.

27. Kanaya S, Haruta S, Kawamura Y, et al. Video: laparoscopy distinctive technique for suprapancreatic lymph node dissection: medial approach for laparoscopic gastric cancer surgery. Surg Endosc 2011;25:3928-9.

28. Lee SW, Tanigawa N, Nomura E, et al. Benefits of intracorporeal gastrointestinal anastomosis following laparoscopic distal gastrectomy. World J Surg Oncol 2012;10:267.

29. Nashimoto A, Akazawa K, Isobe $Y$, et al. Gastric cancer treated in 2002 in Japan: 2009 annual report of the JGCA nationwide registry. Gastric Cancer 2013;16:1-27.

30. Siewert JR, Sendler A. The current management of gastric cancer. Adv Surg 1999;33:69-93. 